\title{
Algumas Considerações Sobre Ensino e Aprendizagem na Disciplina Laboratório de Eletromagnetismo
}

Considerations About Learning and Teaching in the Electromagnetism Laboratory

\author{
J. Humberto Dias da Silva \\ Faculdade de Ciências, Departamento de Física \\ Universidade Estadual Paulista, CEP 17033-360, Bauru, SP
}

Recebido em 8 de maio, 2002. Aceito em 26 de setembro, 2002.

\begin{abstract}
Baseados por um lado em avanços conceituais recentes das pesquisas em ensino de ciências, e por outro em nossa experiência docente, propomos algumas reflexões sobre as práticas de ensino e aprendizagem na disciplina Laboratório de Eletromagnetismo. Apresentamos e interpretamos resultados e comentários dos estudantes, os quais forneceram elementos para a análise da efetividade de propostas de novo enfoque para esta disciplina.
\end{abstract}

Based on recent research in science education, and on teaching experience, we analyze the learning and teaching process in the Electromagnetism laboratory for undergraduate physics students. Data and reasoning obtained from the answers of some groups of students are used to help the analysis of the effectiveness of the changes proposed in this physics laboratory.

\section{Introdução}

Professores e pesquisadores da área de ensino de ciências geralmente imputam grande importância ao espaço de aprendizado de física ocorrido nas disciplinas experimentais $[1,2,3]$. Aparentemente também existe um consenso entre docentes e estudantes de que deve haver mudanças na maneira tradicional de se enfocar estas disciplinas [1,4-7]. Apesar disto há relatos de que esta importância declarada algumas vezes é mais questão de postura que de prática efetiva $[5,8]$, e também há observações críticas a respeito do real benefício que estas disciplinas possam trazer ao processo de aprendizado dos estudantes [9].

A disciplina Laboratório de Eletromagnetismo é a primeira disciplina experimental da parte específica que os estudantes dos cursos de Física realizam. Nela, em princípio, os estudantes têm espaço propício para realizar experimentos um pouco mais detalhados sobre o Eletromagnetismo Clássico. Os experimentos geralmente apresentam um maior grau de complexidade em relação aos experimentos das disciplinas básicas. Esta escolha de maior complexidade neste ponto do curso baseia-se na hipótese, nem sempre correta, de que o grau de maturidade e entendimento do estudante podem alcançar esta complexidade [6,10]. Esta é uma questão que merece cuidado, pois corremos o risco de colocar o estudante em um emaranhado experimental, contribuindo para afastar dele o entendimento mais profundo sobre os conceitos que queremos trabalhar. Isto é especialmente válido quando tratamos das turmas dos cursos de Licenciatura em Física, pois além de se defrontarem com questões relativas ao conteúdo específico, estes estudantes também estão vivenciando procedimentos práticos de ensino em disciplinas experimentais. A tendência de reproduzir estes procedimentos práticos posteriormente com seus futuros alunos é grande.

Baseados por um lado em avanços conceituais recentes das pesquisas em ensino de ciências, e por outro em nossa experiência docente junto aos estudantes do Curso de Licenciatura em Física da Unesp - Bauru, convidamos docentes e estudantes de Licenciatura em Física, para refletirmos sobre alguns aspectos relacionados aos processos de ensino e aprendizagem na disciplina Laboratório de Eletromagnetismo, tentando analisar o que poderia ser mudado nestas práticas para que haja melhor aproximação entre seus objetivos de aprendizagem e os resultados práticos observados.

\section{Repensando alguns aspectos}

Nas pesquisas realizadas durante as três últimas décadas sobre o aprendizado de ciências pode-se notar consenso sobre pontos importantes pertinentes ao processo. Um destes pontos de consenso é a importância de entender o aprendizado em termos de desenvolvi- 
mento conceitual, em vez de simples acréscimo de novas informações [11], ou de simples substituições de concepções existentes por novas [12]. Desenvolvimentos mais recentes [2,5] consistem em resgatar algumas propostas pioneiras sobre o tema [3] dando um enfoque investigativo às práticas de laboratório.

Estas pesquisas apresentam fortes implicações para as práticas em sala de aula por mudarem a concepção, adotada muitas vezes, de que as práticas de laboratório devam ser meras ilustrações da teoria. Quando abordados por este prisma os experimentos realizados adquirem uma orientação rígida e dogmática [2], na qual o critério de verdade é por excelência o da autoridade da teoria, do docente, e do livro-texto. Segundo este ponto de vista os experimentos são usados para verificar se existe ou não uma concordância dos resultados com a teoria, e, não raro, quando existe a negativa, afirma-se que o experimento deve ser repetido até que se consiga o resultado "adequado".

Em contraposição a algumas propostas de que os experimentos neste nível devam necessariamente implicar em um alto grau de complexidade, observamos que, neste caso, a probabilidade de termos um enfoque tecnicista do problema apresentado é grande, conforme apontado por Salinas [2], Sebastia [4] e White [9]. Os objetivos desta disciplina não são prioritariamente técnicos. Preferimos a proposta de Nedelsky [3] de que a função central dos laboratórios de física é dar ao estudante a oportunidade de explorar os vários aspectos da relação entre a descrição física da realidade e a própria realidade, acrescida dos aspectos motivacionais a nível de experiência pessoal e de trabalho em grupos $[8,9,10]$.

Quando os experimentos são demasiadamente complexos não há tempo para o estudante refletir e analisar em detalhe o problema proposto. Nestes casos os grupos de estudantes acabam passando diretamente à execução do experimento, a qual é geralmente descrita em etapas bem definidas pelo professor, sem antes ter uma noção clara do seu propósito. Os aspectos negativos deste tipo de prática também são observados por outros pesquisadores $[4,6,9]$. Neste caso jargões do tipo "o experimento mostrou-se de acordo com a teoria", sem que nenhum espaço para levantar hipóteses tenha sido propiciado, muito pouco acrescentam ao entendimento dos estudantes sobre os aspectos físicos e também sobre o aspecto da natureza da ciência que está sendo "testada".

Desta maneira, podemos considerar que os principais quesitos para escolher os problemas experimentais a serem abordados são aqueles de proporcionar o adequado tema e o adequado nível de hipóteses a serem testadas, de acordo com o estágio de desenvolvimento cognitivo, e com o interesse dos estudantes.

\section{Uma proposta baseada no processo investigativo}

A partir das considerações sobre os problemas levantados acima, foi possível elaborar uma proposta para a disciplina Laboratório de Eletromagnetismo do curso de Licenciatura em Física da Unesp-Bauru. Nesta proposta cada experimento foi dividido em três etapas. Na primeira etapa um problema aberto, tema do experimento, é apresentado aos estudantes a fim de promover sua reflexão sobre o tema. O resultado das idéias que surgem durante esta reflexão é analisado, em classe e em reflexão do professor após a aula. No segundo momento uma proposta de experimento, elaborada pelo professor sobre o mesmo tema, é apresentada aos estudantes. Nesta proposta levamos em consideração, e tentamos propor como hipóteses, algumas idéias levantadas pelos estudantes no momento anterior. A análise dos resultados, a exposição das discussões, e o fechamento de algumas conclusões sobre o experimento proposto pelo professor, é realizada em sala, na última etapa.

Nesta proposta, os aspectos de investigação do trabalho de laboratório no sentido proposto por Gil Perez [5] são enfatizados. Resumimos os principais pontos da proposta em:

i) Propor situações-problema em vez de experimentos fechados.

ii) Analisar, aproveitar, e valorizar as propostas dos estudantes, colocando-as quando possível como hipóteses a serem testadas.

iii) Não menosprezar nesta disciplina o potencial didático de experimentos semi-quantitativos que envolvam a aplicação prática direta de conceitos, propiciando terreno adequado para o desenvolvimento conceitual e das habilidades dos estudantes.

iv) Permitir que os estudantes tenham propostas sobre a maneira de encarar o problema físico e de executar os experimentos.

v) Propiciar cooperação e integração dos grupos na solução dos problemas apresentados e na analise coletiva dos resultados obtidos.

\section{Exemplos de aplicação}

Para ilustrar, vamos apresentar aqui dois experimentos realizados na disciplina durante o primeiro semestre de 2002. Apresentamos os experimentos com pequeno grau de orientação propositalmente, para não incorrer no erro de propor experimentos totalmente direcionados. Sob este ponto de vista vamos analisar que benefícios experimentos quantitativos ou semiquantitativos podem apresentar aos estudantes.

No primeiro exemplo propusemos um experimento para medir o momento magnético de uma barra imantada. Alguns pontos sobre a aplicabilidade que o conhecimento do momento magnético possa ter no cotidiano dos estudantes foram salientados. Como ilustração citamos a questão do dimensionamento dos alto falantes 
de equipamentos sonoros, e as cabeças de gravação de fitas e cartões magnéticos. Nenhum equipamento é trazido ao laboratório, apenas a pequena barra cilíndrica, objeto da proposta. Assim os estudantes são confrontados com o problema, e após um intervalo geralmente pequeno de maturação, algumas propostas começam a surgir, e desencadeiam um interessante processo de propostas onde os estudantes acabam mostrando muitas de suas concepções a respeito do tema. Estas concepções podem ser trabalhadas no momento em que são expressas e também na aula seguinte. Nesta etapa a interação que ocorre nos grupos é fundamental para o processo. A partir das questões levantadas pelos grupos, o professor atento pode expor em sala ricos aspectos associados à historia da ciência, ao conhecimento formal, e a aplicações práticas da disciplina. Algumas vezes isto é difícil de realizar no instante em que as questões aparecem. É importante que os pontos levantados pelos grupos também sejam considerados pelo docente durante a semana que precede a realização do experimento, para serem retomados em melhores condições na aula seguinte.

As propostas dos estudantes para realização de experimentos para medir o momento magnético de uma barra foram interessantes. Por exemplo, podemos citar a proposta de uma equipe que sugeriu a colocação da barrinha em ângulo com o campo de um grande imã em forma de U, em um eixo perpendicular ao campo, para realizar movimento oscilatório. $\mathrm{O}$ momento magnético seria determinado a partir da freqüência de oscilação.

No esquema, reservamos o segundo par de aulas para tentar promover coletivamente uma evolução das representações anteriormente apresentadas. Isto foi feito partindo dos conceitos apresentados pelos estudantes, enfatizando na proposta de experimento os pontos que apresentaram conceitos a serem trabalhados. Uma das possibilidades é introduzirmos alguns dos conceitos como hipóteses de verificação na proposta dos experimentos [5], de maneira que a mudança conceitual possa ser trabalhada de forma mais natural, sem necessariamente enfocarmos o conflito entre a representação prévia do estudante e o conceito aceito dentro da teoria estudada. Isto propicia um caráter de investigação ao processo de aprendizagem, no sentido mais diretamente proposto por Gil Perez [5], mas que também é apontado como altamente benéfico por outros autores [2-4,6,9].

Propusemos aos estudantes o experimento quantitativo clássico da medida do momento magnético de uma barra usando uma balança de torção e um solenóide. Neste experimento um campo magnético causado por corrente contínua que percorre o solenóide provoca um pequeno torque na barra, o qual é equilibrado pelo torque mecânico de uma balança de torção. Com a devida calibração e a consideração de fatores como o campo magnético terrestre, o comprimento (finito) do solenóide, e a correta triangulação para obtenção do ângulo de equilíbrio, o experimento pode ser realizado com um erro em torno de $2 \%$.

Neste experimento ocorreram dificuldades de visu- alização por parte dos estudantes. A principal dificuldade foi identificar corretamente os vários ângulos que devem ser usados na execução do experimento. Há ângulos envolvidos na calibração da balança, entre o eixo do solenóide e o eixo do imã, o ângulo de deflexão da balança ao aplicar o campo magnético, e o ângulo interno do solenóide, quando consideramos a aproximação de solenóide finito. Houve dificuldades também na determinação do ângulo de deflexão a partir de triangulação do feixe de laser. Com esta complexidade o tempo de duas horas foi escasso para a realização do experimento e algumas equipes optaram por retornar em horários diversos para complementarem ou tomarem novos dados. Um detalhe importante, quando o tempo permite, é numerar algumas barras e pedir para que os vários grupos meçam cada uma delas, identificando-as com as medidas. Isto é importante por possibilitar a comparação entre os dados dos vários grupos, e permitir que o teste das hipóteses seja feito com troca de informações e debates intermediados entre grupos. Assim, os estudantes encontram espaço, inexistente na maioria das propostas, para explicar suas hipóteses e debater suas idéias coletivamente. Este processo é rico e contribui de maneira significativa para o sucesso da proposta.

Apesar das propostas e discussões interessantes ocorridas, o resultado da análise neste experimento muitas vezes se perdeu na complexidade técnica dos dados. Por este motivo optamos aqui por analisar em maior detalhe os resultados obtidos no experimento semi-quantitativo, apresentado a seguir. Não propomos com isto o abandono de propostas quantitativas. Porém é importante não enfatizar demasiadamente os aspectos quantitativos dos experimentos em detrimento de outros aspectos igualmente importantes relativos ao aprendizado.

Para citar um experimento semi-quantitativo utilizado na disciplina, recorremos à determinação de superfícies equipotenciais e campos elétricos em geometrias diversas.

O problema colocado foi: Como os campos elétricos podem ser medidos na prática? Que experimento você faria para medir os campos elétricos e mapear as superficies equipotenciais?

Vejamos inicialmente algumas propostas feitas pelos estudantes:

Grupo A - “colocamos um dipolo elétrico conhecido num campo elétrico cujo módulo desejamos saber. Através do torque provocado no dipolo, podemos calcular o módulo de $\boldsymbol{E}$. Isto pode ser feito usando uma balança de torção, de maneira análoga ao experimento anterior".

Comentário: esta proposta indica que o experimento sobre momento magnético fez parte da bagagem dos estudantes no experimento seguinte. Uma interessante associação entre o momento magnético da barra e o momento de dipolo elétrico foi realizada, sem que nenhuma menção formal tivesse sido feita pelo professor. Embora a proposta seja correta do ponto de vista 
conceitual, os estudantes não perceberam que ela seria de difícil realização prática, pois não se pode manter facilmente dipolos elétricos para servirem de prova, contrariamente ao que ocorre com os dipolos magnéticos. Além disso a proposta aparentemente não leva em consideração a variação espacial do campo, e o conseqüente esforço de tomada de dados que teria que ser empregado para um mapeamento adequado do campo. Estes aspectos práticos negativos não diminuem entretanto o interesse da proposta.

Grupo B - "criar um campo elétrico uniforme em uma determinada região. Isso pode ser feito através de placas paralelas" (apresentam figura de geometria de placas paralelas, e duas cargas negativas colocadas em plano paralelo às placas, com linhas de campo corretamente desenhadas). "Se colocarmos duas cargas puntiformes iguais a uma distância $l$ da placa carregada positivamente, haverá uma força exercida pelo campo elétrico $\boldsymbol{E}$ nas duas cargas e estas receberão uma certa aceleração e velocidade como mostra a figura". Ilustraram com vetores $\mathbf{F}$ e $\mathbf{v}$ colocados em uma das cargas negativas e dirigidos para a placa positiva.

Comentário: notamos que duas cargas foram utilizadas, provavelmente para denotar a constância do campo. Observamos que aqui também a constância do campo foi assumida. Não explicitaram como as grandezas mencionadas seriam medidas.

Grupo C - Apresentam uma figura onde a parte externa da esfera de um gerador Van de Graaff é ligada a uma placa plana que se encontra numa cuba com óleo e grãos de farinha. Existe outra placa paralela na cuba, porém nada está conectado a esta. As placas estão a uma distância maior que seu comprimento. Linhas de campo (não uniforme) foram desenhadas corretamente, porém as equipotenciais foram desenhadas como linhas paralelas às placas. "liga-se o positivo do Van de Graaff em um dos eletrodos. Basicamente com isto pode-se ver as linhas de campo, e assim, já é possivel imaginar as superficies equipotenciais sabendo-se que as mesmas são perpendiculares às linhas do campo elétrico. Podemos perceber que o campo elétrico é mais intenso na região onde há maior concentração dos grãos de farinha, ou seja, próximo aos eletrodos. Nisso tudo considerando que os grãos de farinha não adquirem carga no contato com o líquido (óleo). Uma possível medida para o campo elétrico seria tirar uma foto da formação das linhas de campo e contar os grãos de farinha numa determinada região, e com isso calcular a densidade por centímetro cúbico. A mesma deverá diminuir à medida que nos afastamos das placas".

Comentário: os estudantes utilizaram um experimento demonstrativo apresentado a eles no laboratório básico (Laboratório de Física Geral IV), e acrescentaram o interessante processo de fotografar e medir a densidade de grãos para estimar o valor do campo. A proposta é interessante o suficiente para possível utilização como hipótese a ser investigada no experimento proposto pelo professor. Infelizmente não dispomos dos equipamentos fotográficos necessários para promover a comparação entre os valores de campo determinados desta maneira e os valores medidos no experimento. Nota-se a contradição entre a afirmação de que as equipotenciais deveriam ser perpendiculares ao campo com o desenho das equipotenciais paralelas às placas. Esta foi uma das raras propostas que levaram em conta variações espaciais do campo, enquanto nos outros grupos foi observada uma tendência clara de não se levar em conta estas possíveis variações espaciais.

$\mathrm{Na}$ aula seguinte, correspondente à segunda etapa do experimento, propusemos um experimento para o mapeamento das superfícies equipotenciais utilizando uma cuba com água, dois eletrodos de formas a escolher (pelo menos três pares), uma fonte para polarizar, e um voltímetro digital para medir as diferenças de potencial. Algumas instruções foram dadas para garantir um mapeamento adequado das equipotenciais. $\mathrm{O}$ intuito era propiciar condições para que os estudantes "vissem" o campo a partir das equipotenciais, tendo a possibilidade de testar vários aspectos presentes nas suas concepções. Na seqüência apresentamos alguns resultados das interpretações dos grupos:

Grupo A - Relataram que o campo deveria ser paralelo ao eixo que une uma placa a uma carga circular pequena (identificada por eles como puntiforme), apesar de terem calculado o campo utilizando as distâncias perpendiculares às equipotenciais.

Grupo B - Esperavam que as superfícies equipotenciais para a simulação de um dipolo fossem esferas concêntricas com os centros dos polos.

Grupo C - Utilizaram na determinação dos campos apenas as linhas centrais de simetria nas três geometrias escolhidas (duas placas, placa e ponto, dois pontos).

Em nossa opinião o principal ponto relacionado ao conhecimento específico deste experimento foi tratar com os estudantes o conceito de gradiente, e a conseqüente ligação entre potencial e campo elétrico. Analogias com situações de mapeamento topográfico de relevos, levou-os a um melhor entendimento das curvas equipotenciais, permitindo que calculassem o módulo e indicassem corretamente a direção e o sentido do campo usando as equipotenciais traçadas. Antes desta fase, os estudantes citam a relação $\mathbf{E}=$ $\operatorname{grad}(\mathrm{V})$ no corpo do relatório, porém pouquíssimas vezes conseguiram indicar, a partir das equipotenciais obtidas na prática, uma maneira de calcular $\mathbf{E}$ usando esta relação. Isto quer dizer que o docente que proponha que o campo elétrico seja calculado usando esta relação em um roteiro de experimento, por melhor intencionado que seja, está perdendo uma grande oportunidade de deixar que o estudante aprofunde seus conhecimentos, pensando ele próprio esta maneira de encarar o problema.

Este último experimento não apresenta as vantagens quantitativas do primeiro, e talvez alguns professores possam achá-lo simples demais para um Laboratório de Eletromagnetismo do terceiro ano. Porém em troca do aspecto quantitativo podemos citar algumas qualidades. Em primeiro lugar o único equipamento necessário 
à sua realização é um voltímetro, que atualmente pode ser adquirido a baixo custo por uma escola ou que talvez possa ser encontrado entre as ferramentas de trabalho de um estudante com pai eletricista. O experimento pode ser realizado utilizando-se como fonte de tensão uma bateria de $9 \mathrm{~V}$, ou um conjunto de pilhas de lanterna. Através das linhas equipotenciais bidimensionais traçadas o estudante pode descobrir a relação entre potencial e campo elétrico, exemplificando este último com uma maneira concreta, até então provavelmente desconhecida. Colocados em questão antes do experimento se o campo elétrico tinha uma existência real ou se era mera abstração, os estudantes, mesmo neste grau, ficaram em sua maioria indecisos, e optaram muitas vezes por considerar que o campo era apenas uma entidade "teórica" que não conseguiríamos expressá-lo de uma maneira prática. Entre os que responderam que o campo possuía uma existência real, muitos descreveram o experimento com cargas para testá-lo "soltase uma carga com massa conhecida e mede-se a aceleração". Porém quando indagados a respeito de como seria possível saber se o campo seria constante ou não naquela região do espaço, nenhum fez menção a alguma maneira de mapear o campo. Segundo nosso entendimento este experimento, embora semi quantitativo, apresenta um real valor no contexto da disciplina por ilustrar de maneira metodologicamente simples uma grandeza física muito importante para a formação do estudante.

\section{Comentários dos estudantes sobre a proposta}

Durante o desenvolvimento da disciplina os estudantes fizeram vários comentários sobre as modificações propostas para a disciplina. Com o intuito de entender como a proposta foi recebida pelos estudantes, apresentamos aqui alguns pontos que consideramos importantes em seus comentários.

Ao conhecer a proposta da disciplina e nos primeiros experimentos houve dúvidas quanto ao que se pretendia que fosse relatado, e quanto ao critério que seria usado nas correções dos trabalhos, em alusão clara à preocupação com relação aos critérios de avaliação a serem adotados.

Durante a realização dos primeiros experimentos houve um desconforto inicial em relação aos procedimentos a serem utilizados nos experimentos propostos pelo professor, pois apesar de que os objetivos, e até certo ponto os mecanismos principais a serem considerados foram estabelecidos, outros pontos foram deixados em aberto no experimento, entre eles a metodologia de aquisição e interpretação dos dados.

Estas dificuldades iniciais foram superadas durante o decorrer do semestre, e em nosso entender contribuíram para desenvolver uma maior autonomia dos estudantes frente a situações experimentais. Conforme apontado por Barolli [8], a superação das dificuldades iniciais e os avanços da autonomia observados, podem ser atribuídos em parte à melhor estruturação dos grupos com o decorrer do tempo de interação. Esta melhor estruturação pode auxiliar no estabelecimento de ações cooperativas que contribuem para a superação das dificuldades práticas.

A possibilidade de aproveitar melhor suas próprias idéias foram pontos importantes sobre os quais os estudantes fizeram comentários em várias oportunidades manifestando clara aprovação. Em nossa avaliação este é um dos principais pontos favoráveis da proposta: propicia motivação e envolvimento dos estudantes no sentido de utilizar os conhecimentos disponíveis para abordar situações práticas. Trocas de informações entre os membros de cada grupo e entre os grupos da turma, e a intermediação do professor foram favorecidas de maneira natural nesta dinâmica.

Uma dificuldade não claramente percebida pelos estudantes, mas externada em seus comentários em várias ocasiões, foi a de considerar a proposta do professor como sendo a única "correta" e de tentar avaliar a validade ou não de sua proposta pela semelhança com a proposta do professor. Tal postura dos estudantes é um problema real deste tipo de proposta e necessita uma atenção especial do professor que se propõe a implementá-las. Esta é uma conseqüência negativa de metodologias tradicionalmente colocadas para o estudante, e que merece intervenções cuidadosas do professor.

Apesar da grande importância imputada por nós às propostas dos estudantes, o envolvimento posterior dos grupos com suas próprias propostas não foi grande. Atribuímos isto em parte à dificuldade mencionada no parágrafo anterior e também à facilidade dos estudantes em modificarem suas propostas a partir do conhecimento de novos dados, ou de objeções consistentes que possam ser levantadas sobre seus argumentos.

\section{Considerações finais}

Nesta abordagem do Laboratório de Eletromagnetismo os estudantes tiveram a oportunidade de fazer uma proposta de experimento, de analisar os resultados de experimento proposto pelo professor, e de fazer uma análise comparativa entre sua proposta de experimento e aquele proposto pelo professor. Entretanto, o experimento realizado ainda não é verdadeiramente uma investigação proposta pelo grupo de estudantes, e sim uma investigação dirigida. Apesar disto existe espaço para que alguns aspectos propostos diretamente pelos estudantes sejam enfocados: se o experimento proposto pelo professor contiver hipóteses de verificação relacionadas às propostas às representações prévias dos estudantes, muitas das quais manifestaram-se na etapa inicial de proposição, um dos importantes objetivos da disciplina será atingido [3,5].

Nesta disciplina é importante termos em conta que, em muitas situações, a inadequada complexidade dos experimentos propostos associada ao pequeno 
tempo dedicado à reflexão sobre os conceitos e interpretação dos resultados, pode infelizmente levar a poucos avanços na direção desejada, ou seja ao efetivo aprofundamento do entendimento dos conceitos por parte dos estudantes. Este é um aspecto negativo, pois além do entendimento específico da disciplina os estudantes de licenciatura estão vivenciando exemplos dos processo de ensino e aprendizagem e dos relacionamentos em grupo $[8,9,10]$ aplicados à solução de situações práticas

O papel dos grupos, o qual já tem sua importância em abordagens tradicionais de disciplinas de laboratório, é fundamental para o sucesso deste tipo de proposta. A interação entre os membros de cada grupo e entre grupos foi especialmente produtiva durante as proposições dos estudantes. Neste momento houve uma forte interação entre os membros de cada grupo, e uma abertura muito maior que a normalmente observada para a mediação buscada junto ao professor. A mediação do professor ocorreu principalmente quando algum membro propunha um experimento ao grupo e tentava explicar baseado em seu entendimento dos conceitos físicos. As propostas e justificativas geralmente causavam questionamentos, troca e confronto de idéias com os colegas. Isto também promoveu de maneira natural o papel do professor como mediador das discussões.

A dificuldade rotineira de contarmos com menos tempo do que gostaríamos foi potencializada nesta proposta. Para tratar da profusão de idéias, que ocorre quando deixamos um problema aberto para consideração dos grupos, é necessário um intervalo de tempo geralmente não disponível nas propostas tradicionais. Para tentar superar esta dificuldade foi adotado o procedimento de deixar uma semana para cada uma das três etapas: propostas, realização, e discussões (a disciplina é oferecida em duas horas semanais). Isto implica que um menor número de experimentos pode ser tratado (em geral 4 a 5 por semestre!). Porém o fato de termos realizado menos experimentos não significa que tenhamos abordado um número menor de situações práticas e tratado menos temas experimentais, aliás, tem-se a impressão clara de que ocorre justamente o oposto. Pelas próprias respostas e atitudes dos estudantes, nos parece que com esta abordagem estamos mais próximos dos objetivo do laboratório didático: promover a oportunidade ao estudante de confrontar seus conceitos sobre a física com o resultado dos experimentos, e de repartir suas concepções com os outros estudantes e com o professor $[5,8,9,10]$.

$\mathrm{O}$ fato de podermos abranger os itens principais da proposta em maior ou menor grau em cada experimento, justifica a diminuição do número de experimentos que podem ser trabalhados na disciplina pelo aumento da efetividade em que cada tópico é trabalhado. Além disso, pela riqueza de troca de experiência que proporcionam, podemos dizer que ao invés de reduzir o número de temas abordados (com a redução do número de experimentos) o número de temas abordados na disciplina é ampliado, pois há espaço para se discutir aspectos históricos, aplicações e abrangência dos conceitos.

Longe de representar uma proposta fechada, este conjunto de idéias é um convite a docentes e estudantes de licenciatura a refletirem sobre o aprendizado no Laboratório de Eletromagnetismo.

\section{Agradecimentos}

Agradeço aos estudantes do curso de Licenciatura em Física da Unesp - Bauru, e aos Professores Elizabeth Barolli e Fernando Bastos, pelas interessantes discussões, ao Mestre Júlio César Ribeiro, cujo trabalho de orientação me levou a repensar minhas próprias práticas de ensino, e a dois estimados professores de física experimental Jorge Ivan Cisneros e Curt Egon Hennies, do IFGW - Unicamp, que me propiciaram, enquanto estudante, o gosto pelas disciplinas experimentais.

\section{Referências}

[1] D. Gil Perez e M. G. Ozamis, Ensenãnza de Las Ciencias y la Matemática, Editorial Popular S.A / Ministério de Educacion y Ciencia, Espanha, 1992.

[2] J. S. Salinas, L.C. de Cudmani, Revista de Enseñanza de la Física, 5, 10 (1992).

[3] L. Nedelsky, Am. J. Phys. 26, 51 (1958).

[4] J.M. Sebastia, Enseñanza de Las Ciencias, 5, 196 (1987).

[5] D. Gil Perez, P. Valdés Castro. Enseñanza de las Ciências, 14, 155 (1996).

[6] M. E. Gonzales, Enseñanza de las Ciências, 10, 206 (1992).

[7] J.C. Ribeiro, O Papel do Professor no Laboratório de Física Básica: Um Exemplo Ilustrativo, Dissertação de Mestrado, Unesp-Bauru (2000).

[8] E. Barolli, Reflexões sobre o Trabalho dos Estudantes no Laboratório Didático, Tese de Doutoramento, USPSP, (1998)

[9] R.T. White, Int. J. Sci. Educ., 18, 76 (1996).

[10] E. Barolli e A. Villani, Ciência e Educação, 6, 1 (2000).

[11] A Villani, Revista Brasileira de Ensino de Física, 6, 76 (1984).

[12] E.F. Mortimer, Investigações em Ensino de Ciências, 1, 22 (1996). 